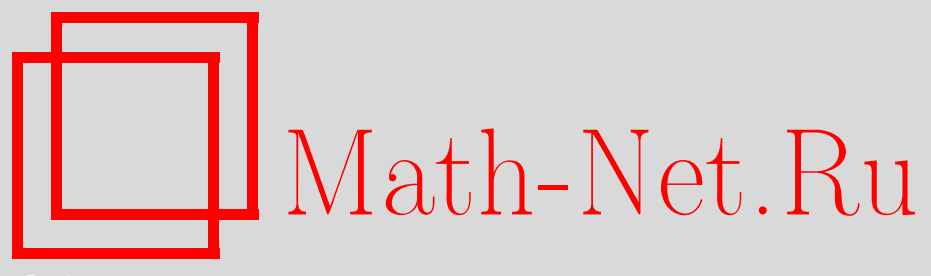

А. С. Костенко, О подобии самосопряженному некоторых $J$-неотрицательных операторов, Матем. заметки, 2006, том 80, выпуск 1, 135-138

DOI: https://doi.org/10.4213/mzm2789

Использование Общероссийского математического портала Math-Net.Ru подразумевает, что вы прочитали и согласны с пользовательским соглашением http://www . mathnet.ru/rus/agreement

Параметры загрузки:

IP : 3.85 .7 .115

26 апреля 2023 г., 02:19:27

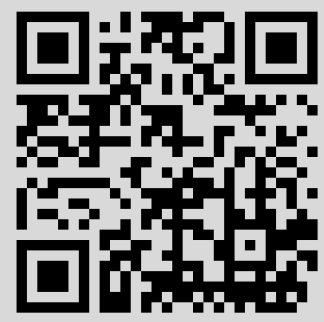




\section{О ПОДОБИИ САМОСОПРЯЖЕННОМУ НЕКОТОРЫХ $J$-НЕОТРИЦАТЕЛЬНЫХ ОПЕРАТОРОВ}

\section{А. С. Костенко}

1. Введение. Пусть $\omega: \mathbb{R} \rightarrow \mathbb{R}_{+}-$неотрицательная функция на $\mathbb{R}$ такая, что $\omega(\cdot) \in$ $L_{\mathrm{loc}}^{1}(\mathbb{R})$ и $\omega(x)>0$ для почти всех $x \in \mathbb{R}$. Обозначим через $L^{2}(\mathbb{R}, \omega)$ гильбертово пространство измеримых функций, квадратично суммируемых на оси с весом $\omega(x)$; внутреннее произведение функций $f, g \in L^{2}(\mathbb{R}, \omega)$ определяется обычным образом: $(f, g)_{\omega}=$ $\int_{\mathbb{R}} f(t) \overline{g(t)} \omega(t) d t$. Рассмотрим в $L^{2}(\mathbb{R}, \omega)$ линейный оператор

$$
L_{\omega}=-\frac{\operatorname{sgn} x}{\omega(x)} \frac{d^{2}}{d x^{2}}, \quad \operatorname{dom}(L)=\left\{f \in L^{2}(\mathbb{R}, \omega): f, f^{\prime} \in W_{1, \mathrm{loc}}^{1}(\mathbb{R}), L_{\omega} f \in L^{2}(\mathbb{R}, \omega)\right\} .
$$

В заметке изучается вопрос о подобии самосопряженному оператора $L_{\omega}$. Напомним, что операторы $A_{1}$ и $A_{2}$, действующие в $B$-пространстве $X$ с областями определения $\operatorname{dom}\left(A_{1}\right)$ и $\operatorname{dom}\left(A_{2}\right)$ называют подобными, если найдется ограниченный и ограниченно обратимый оператор $T$ в пространстве $X$ такой, что $T\left(\operatorname{dom}\left(A_{1}\right)\right)=\operatorname{dom}\left(A_{2}\right)$ и $A_{2}=T A_{1} T^{-1}$.

Впервые подобие несамосопряженного оператора $L_{w}$ вида (1) самосопряженному было установлено Б. Чургусом и Б. Найманом [1] (случай $\omega(x)=1)$, а также А. Фляйге и Б. Найманом [2] $\left(\omega(x)=|x|^{\alpha}, \alpha>-1\right)$ при помощи теории Крейна-Лангера дефинизируемых операторов в пространствах Крейна. Затем эти результаты обобщались во многих работах (см. [3]-[7], а также библиографию в них). Так, в работах [3] и [5] получен критерий подобия самосопряженному или нормальному оператору в терминах величины взаимодействия $c$ для операторов, задаваемых в $L^{2}\left(\mathbb{R},|x|^{\alpha}\right)$ дифференциальным выражением

$$
l_{c}:=\frac{\operatorname{sgn} x}{|x|^{\alpha}}\left(-\frac{d^{2}}{d x^{2}}+c \delta\right), \quad c \in \mathbb{C} \backslash\{0\} .
$$

В работах М. М. Фаддеева, Р. Г. Штеренберга [6] и И. М. Карабаша, М. М. Маламуда [4] получены достаточные условия подобия самосопряженному для операторов ШтурмаЛиувилля

$$
A_{q}:=\operatorname{sgn} x\left(-\frac{d^{2}}{d x^{2}}+q(x)\right), \quad x \in \mathbb{R},
$$

соответственно с быстроубывающим и конечнозонным потенциалами $q(\cdot)$. Фаддеев и Штеренберг в [7] получили следующее достаточное условие подобия оператора $L_{\omega}$ вида (1) самосопряженному.

Теорема 1 [7]. Пусть $\omega(x)=p(x)|x|^{\alpha}, \alpha>-1$, а функция $p(\cdot)$ локально абсолютно непрерывна на $\mathbb{R}$ вместе со своей первой производной и удовлетворяет двусторонней оченке $0<c \leqslant p(x) \leqslant C<\infty, x \in \mathbb{R}$. Пусть такэе выполнены условия

$$
\begin{gathered}
\int_{\mathbb{R}_{ \pm}} \frac{|x|^{\alpha}\left|p(x)-c_{ \pm}\right|}{\left(|x|^{|\alpha| / 2}+1\right)^{\operatorname{sgn} \alpha}} d x<\infty, \quad c_{ \pm}>0, \\
\int_{\mathbb{R}} \frac{\left|3\left(u^{\prime \prime}(x)\right)^{2} / u^{\prime}(x)-2 u^{\prime \prime \prime}(x)\right|}{\left(u^{\prime}(x)\right)^{2}\left(|u(x)|^{|\alpha| / 2}+1\right)^{\operatorname{sgn} \alpha}} d x<\infty
\end{gathered}
$$

где $и(x)$ определяется следующим образом:

$$
u(x):=\operatorname{sgn} x\left(\left.\left|\frac{\alpha+2}{2} \int_{0}^{x} \sqrt{p(t)}\right| t\right|^{\alpha / 2} d t \mid\right)^{2 /(2+\alpha)} .
$$

Тогда оператор $L_{\omega}$ подобен самосопряженному.

(C) A. C. Костенко, 2006 
Основным результатом данной заметки является теорема 4. Используя теорию Крейна-Лангера дефинизируемых операторов (см. [8]), а также резольвентный критерий Набоко-Маламуда подобия самосопряженному ([9], [10]), мы покажем, что требования на функцию $p(\cdot)$ можно ослабить. А именно, для подобия оператора $L_{\omega}$ самосопряженному достаточно выполнения неравенства (4) и непрерывности функции $p(\cdot)$ в окрестности нуля (см. следствие 1).

2. О струне Крейна. Пусть $\omega_{+}(x):=\omega(x), x>0$. Всюду в дальнейшем будем считать, что $\int_{\mathbb{R}_{+}} t^{2} \omega_{+}(t) d t=\infty$. Рассмотрим в гильбертовом пространстве $L^{2}\left(\mathbb{R}_{+}, \omega_{+}\right)$ минимальный оператор, который соответствует струне Крейна $S$ с плотностью распределения масс $\omega_{+}(x)$,

$$
L_{0}^{S}:=-\frac{d^{2}}{d m(x) d x}, \quad m(x)=\int_{0}^{x} \omega_{+}(t) d t
$$

$\operatorname{dom}\left(L_{0}^{S}\right)=\left\{f \in L^{2}\left(\mathbb{R}_{+}, \omega_{+}\right): f, f^{\prime} \in W_{1, \text { loc }}^{1}\left(\mathbb{R}_{+}\right), f(0)=f^{\prime}(0)=0, L_{0}^{S} f \in L^{2}\left(\mathbb{R}, \omega_{+}\right)\right\}$.

Следуя [4], обозначим через $\varphi(x, \lambda)$ решение уравнения

$$
-\frac{d^{2} u(x)}{d m(x) d x}-\lambda u(x)=0, \quad x>0,
$$

удовлетворяющее начальным условиям $u(0)=1, u^{\prime}(0)=0$. Через $\psi(x, \lambda)$ обозначается решение уравнения (7), удовлетворяющее начальным условиям $u(0)=0, u^{\prime}(0)=1$.

Заметим, что в данном случае существует предел (см. [11])

$$
M_{\omega_{+}}(\lambda):=\lim _{x \rightarrow+\infty} \frac{\psi(x, \lambda)}{\varphi(x, \lambda)}=\lim _{x \rightarrow+\infty} \frac{\psi^{\prime}(x, \lambda)}{\varphi^{\prime}(x, \lambda)}, \quad \lambda \in \mathbb{C} \backslash \mathbb{R}_{+},
$$

называемый коэффициентом динамической податливости струны. Функция $M_{\omega_{+}}(\cdot)$ принадлежит классу Крейна-Стилтьеса, т.е. допускает интегральное представление

$$
M_{\omega_{+}}(\lambda)=\int_{0}^{+\infty} \frac{d \tau_{\omega_{+}}(s)}{s-\lambda}, \quad \lambda \in \mathbb{C} \backslash \mathbb{R}_{+}, \quad \int_{0}^{+\infty} \frac{d \tau_{\omega_{+}}(s)}{1+s}<+\infty .
$$

Здесь $\tau_{\omega_{+}}(\cdot)$ - неубывающая функция, заданная на промежутке $[0,+\infty)$.

Определим $\lambda^{1 /(2+\alpha)}$ как ветвь многозначной аналитической функции на плоскости $\mathbb{C}$ с разрезом вдоль $\mathbb{R}_{-}$, выделяемую условием $(-1+i 0)^{1 /(2+\alpha)}=e^{i \pi /(2+\alpha)}$.

ПредлоЖение 1 [5]. Если $\omega_{+}(x)=x^{\alpha}, \alpha>-1$, то коэффициент динамической податливости $M_{\omega_{+}}(\cdot)$ струны $L_{0}^{S}$ вида (6) равен

$$
M_{\omega_{+}}(\lambda)=M_{\alpha}(\lambda):=\frac{A_{\alpha}}{(-\lambda)^{1 /(2+\alpha)}}, \quad \lambda \in \mathbb{C} \backslash \mathbb{R}_{+},
$$

где $A_{\alpha}:=M_{\alpha}(-1)>0$.

Далее, используя предложение 1 , при помощи стандартных рассуждений мы можем получить следующую теорему.

Теорема 2. Пусть $L_{0}^{S}$ оператор вида (6) $u \omega_{+}(x)=p(x) x^{\alpha}, \alpha>-1$. Если функиия $p(x)$ удовлетворяет условию

$$
\int_{0}^{+\infty} \frac{|t|^{\alpha}\left|p(t)-c_{+}\right|}{\left(|t|^{|\alpha| / 2}+1\right)^{\operatorname{sgn} \alpha}} d t<\infty, \quad c_{+}>0,
$$

с некоторой константой $c_{+}$, то

$$
M_{\omega_{+}}(\lambda)=M_{\alpha}(\lambda)\left(c_{+}+\bar{o}\left(\lambda^{\frac{1+\alpha}{2+\alpha}}\right)\right)=\frac{A_{\alpha}}{(-\lambda)^{1 /(2+\alpha)}}\left(c_{+}+\bar{o}\left(\lambda^{\frac{1+\alpha}{2+\alpha}}\right)\right), \quad|\lambda| \rightarrow 0 .
$$

В дальнейшем функция $M_{\omega_{-}}(\cdot)$ будет обозначать коэффициент динамической податливости струны $S$ с плотностью распределения масс $\omega_{-}(x):=\omega(-x), x>0$. 
3. О подобии оператора $L_{\omega}$ самосопряженному. Пусть $J$ - оператор в $L^{2}(\mathbb{R}, \omega)$ вида

$$
(J f)(x):=\operatorname{sgn} x f(x), \quad x \in \mathbb{R} .
$$

Очевидно, что $J$ - унитарный оператор в $L^{2}(\mathbb{R}, \omega)$. Рассмотрим пространство Крейна $\mathscr{K}=L^{2}(\mathbb{R}, \widetilde{\omega})$ с внутренним произведением $[\cdot, \cdot]_{\widetilde{\omega}}=(J \cdot, \cdot)_{\omega}$. Здесь $\widetilde{\omega}:=J \omega$. Тогда $J-$ это фундаментальная симметрия в пространстве Крейна $\mathscr{K}=L^{2}(\mathbb{R}, \widetilde{\omega})$. Заметим, что

$$
\left[L_{\omega} f, f\right]_{\tilde{\omega}}=\left(J L_{\omega} f, f\right)_{\omega}>0, \quad f \in \operatorname{dom}\left(L_{\omega}\right), \quad f \neq 0 .
$$

Справедлива следующая лемма

Лемма 1. Оператор $L_{\omega}$ вида (1) является положительным дефинизируемым оператором в пространстве Крейна $\mathscr{K}=L^{2}(\mathbb{R}, \widetilde{\omega}) u$

$$
\sigma\left(L_{\omega}\right) \subset \mathbb{R}
$$

ДокАЗАтЕльство. Положительность оператора $L_{\omega}$ в пространстве $L^{2}(\mathbb{R}, \widetilde{\omega})$ вытекает из (13). Для того, чтобы установить дефинизируемость, достаточно доказать, что резольвентное множество оператора $L_{\omega}$ не пусто.

Воспользовавшись тем фактом, что оператор $L_{\omega}$ является почти разрешимым (в смысле [12]) расширением симметрического оператора, можно получить простое описание комплексного спектра оператора $L_{\omega}$. А именно,

$$
\lambda \in \sigma\left(L_{\omega}\right) \backslash \mathbb{R} \quad \text { точно тогда, когда } M_{\omega_{+}}(\lambda)+M_{\omega_{-}}(-\lambda)=0 .
$$

Учитывая тот факт, что $M_{\omega_{ \pm}}(\lambda)$ являются функциями класса $S$, и анализируя их поведение в окрестности вещественной оси, нетрудно доказать существование такого $\lambda_{0} \in \mathbb{C}_{+}$, что $M_{\omega_{+}}\left(\lambda_{0}\right)+M_{\omega_{-}}\left(-\lambda_{0}\right) \neq 0$. Следовательно, $\lambda_{0} \in \rho\left(L_{\omega}\right)$. Теперь соотношение (14) является следствием дефинизируемости $J$-неотрицательного оператора $L_{\omega}$ (см. [8]).

Дефинизируемые операторы в пространствах Крейна обладают спектральной функцией (см. подробности в [8]), свойства которой отличны от свойств спектральной функции самосопряженного оператора лишь в окрестности так называемых критических точек.

Если в окрестности критической точки спектральная функция дефинизируемого оператора ограничена, то такая точка называется регулярной. В противном случае эта точка называется сингулярной критической точкой. Критическими точками неотрицательных дефинизируемых операторов могут быть только нуль и бесконечность.

Известно (см., например, [3]), что для неотрицательных дефинизируемых операторов с тривиальным ядром подобие самосопряженному эквивалентно регулярности его критических точек.

Вопросу о регулярности критической точки " $\infty$ " посвящено много работ (см. [13] и приведенную там библиографию). Нам понадобится следующий факт (см. теорему 3.6 , а также замечание 3.3 в [13]).

Tеорема 3 [13]. Пусть оператор $L_{\omega}$ имеem вид (1), $\omega \in L_{\text {loc }}^{1}(\mathbb{R}) u \omega(x)>0$ для почти всех $x \in \mathbb{R}$. Если функция $\omega(\cdot)$ абсолютно непрерывна в некоторой проколотой окрестности нуля $B_{0}$, и существуют числа $s_{+}>0$ u $s_{-}>0, s_{ \pm} \neq 1$, такие, что

$$
\lim _{ \pm x \rightarrow+0} \frac{\omega(x)}{\omega\left(s_{ \pm} x\right)} \neq s_{ \pm}, \quad\left(\frac{\omega(x)}{\omega\left(s_{ \pm} x\right)}\right)^{\prime} \in L^{\infty}\left(B_{0}^{ \pm}\right),
$$

(1) 
Отметим, что нуль действительно является критической точкой оператора $L_{\omega}$, так как $x \notin L^{2}\left(\mathbb{R}_{ \pm}, \omega_{ \pm}\right)$и, следовательно, нуль принадлежит непрерывному спектру оператора $L_{\omega}$.

Используя теорему 3, спектральное представление неотрицательных дефинизируемых операторов, а также резольвентный критерий подобия Набоко-Маламуда [9], [10], мы выводим из теоремы 2 следующее достаточное условие регулярности критической точки нуль.

Представим функцию $\omega \in L_{\mathrm{loc}}^{1}(\mathbb{R})$ следующим образом:

$$
\omega(x):=\left\{\begin{array}{ll}
p(x)|x|^{\alpha_{+}}, & x>0, \\
p(x)|x|^{\alpha_{-}}, & x<0,
\end{array} \quad \alpha_{ \pm}>-1 .\right.
$$

Теорема 4. Пусть оператор $L_{\omega}$ определен соотношениями (1) u (17), а функиия $\omega(\cdot)$ удовлетворяет условиям теоремы 3. Тогда для регулярности критической точки нуль, а значит и для подобия оператора $L_{\omega}$ самосопряженному, достаточно, чтобъ

$$
\int_{1}^{+\infty}|t|^{\alpha_{+} / 2}\left|p(t)-c_{+}\right| d t<\infty, \quad \int_{-\infty}^{-1}|t|^{\alpha_{-} / 2}\left|p(t)-c_{-}\right| d t<\infty, \quad c_{ \pm}>0,
$$

с некоторыми константами $c_{+} u c_{-}$.

СледСтвиЕ 1. Пусть функиия $\omega(\cdot)$ имеет вид (17), а неотрицательная функиия $p(\cdot)$ непрерывно дифберенцируема в некоторой проколотой окрестности нуля $B_{0}$, удовлетворяет условиям (18) и $p( \pm 0) \neq 0$. Тогда оператор $L_{\omega}$ подобен самосопряженному.

Благодарности. Автор выражает искреннюю благодарность М. М. Маламуду за руководство работой, а также И. М. Карабашу за многочисленные полезные обсуждения и замечания.

Часть этой работы была проделана автором в Ягелонском университете (г. Краков), в связи с чем хочется упомянуть поддержку Фонда королевы Ядвиги.

\section{СПИСОК ЦИТИРОВАННОЙ ЛИТЕРАТУРЫ}

[1] B. Ćurgus, B. Najman, Proc. Amer. Math. Soc., 123 (1995), 1125-1128. [2] A. Fleige, B. Najman, "Nonsingularity of critical points of some differential and difference operators", Oper. Theory Adv. and Appl., 102, Birkhäuser, Basel, 1998, 85-95. [3] И. М. Карабаш, А. С. Костенко, Матем. заметки, 74:1 (2003), 127-131. [4] И. М. Карабаш, М. М. Маламуд, Докл РАН, 395:3 (2004), 303-307. [5] А. С. Костенко, Матем. заметки, 78:1 (2005), 147-151. [6] М. М. Фаддеев, Р. Г. Штеренберг, "О подобии некоторых сингулярных дифференциальных операторов самосопряженным",Зап. научн. семин. ПОМИ, 270 (2000), 336-349. [7] М. М. Фаддеев, Р. Г. Штеренберг, Матем. заметки, 72 (2002), 292-303. [8] H. Langer, "Spectral functions of definitizable operators in Krein spaces", Functional analysis. Proceedings (Dubrovnik 1981), Lecture Notes in Math., 948, 1982, 1-46. [9] С. Н. Набоко, Функиион. анализ и его прилож., 18:1 (1984), 16-27. [10] М. М. Маламуд, Укр. матем. ж., 37:1 (1985), 49-56. [11] И. С. Кац, М. Г. Крейн, "О спектральных функциях струны", Дополнение II $к$ книге Ф. Аткинсона "Дискретные и непрерывные задачи", Мир, М., 1968. [12] В. А. Деркач, М. М. Маламуд, "Расширение теории эрмитовых операторов и проблема моментов", Анализ 3. Итоги науки и техники. Соврем. матем. и ее прилож, 5, ВИНИТИ, М., 1993. [13] В. Ćurgus, H. Langer, J. Diff. Eqs., 79 (1989), 31-61.

\section{А. С. Костенко}

Поступило

Донецкий национальный университет, Украина

02.01.2006

E-mail: duzer80@mail.ru, duzer@skif.net 